\title{
Electronic Medical Records Software Types in Use within Kisumu County
}

\author{
Rinnie Juma \\ Rongo University \\ Msc Health Informatics Student
}

\author{
James Abila \\ Rongo University Lecturer, \\ Department of Health Informatics \\ and Information Science
}

\author{
John Oluoch \\ Rongo University Lecturer \\ Department of Communication and \\ Information
}

\begin{abstract}
Electronic Medical Records (EMR) refers to a set of computerized interrelated components that enable data collection, storage, and transformation, aggregate and disseminate information about a patient or a client in a health facility. However, EMR Assessment by Kenya National Aids Control Program (NASCOP) and Division of Health Information System, done earlier identified various EMR systems and other software that are supporting the provision of health care in the country. These software were grouped as; Patient Management Software(e.g., C-PADComprehensive Patient Application Data base, OpenMRS-Open Medical Records Software, IQ-CARE and EDPMS), Hospital HMIS Software /ERP Systems, Data Collection and Reporting Software( e.g., C-PAD, OpenMRS, IQ-CARE, EPI-INFO), Data Analysis Software (Kang'a et. al., 2017). This study sought to examine Electronic Medical Records software types in use within Kisumu County. The target population was 525 EMR staff from 7 Sub Counties (clusters) and a sample size of 227 (apportioned to three Sub Counties selected purposively). However, the research intended to reach a sample size of 227 respondents; only 163 out of $227(72 \%)$ questionnaires were received from EMR software users. Data was collected using questionnaires. Descriptive statistics was used to analyze data using Statistical Package for Social Scientist version 22.0.
\end{abstract}

Keywords:- Electronic Medical Records, Software Types.

\section{INTRODUCTION}

Health Care system has had an ongoing focus on improving access to and quality of care, and more recently on cost reduction. However, the primary means to achieve these goals has been to change health care policy, as exemplified by the adoption of health information technology in particular the adoption of patient centered information, characterized by the ability to manage comprehensive patient information. Despite the importance of these systems in health care, little is known about their adoption (Marques et al., 2011).

Studies by Cowan et al., (2019) reveal that "globally, the concept of Electronic Medical Record (EMR) Systems started in the 1970s, but even today there are still many obstacles to physicians' adoption of computer technology. Most EMR packages started as billing systems slowly adding clinical components to gain market share', However, degree of adoption of technologies in health care has been shown to correlate with reduction in mortalities in hospitals.

According to Akanbi et al., (2012), "African nations are still lagging behind developed countries in the availability and use of Electronic Health Records. Most EHRs are sustained by funding from international partnerships raising questions about the sustainability of these systems by the host institutions. Poor infrastructure also increases the cost to set up and maintain the systems"'.

Kang'a et. al., (2017) documents that "Kenya Health Information System (HIS) policy mission is to provide timely, reliable and accessible quality health information for evidence - based decision making to promote the health of the nation. Kenya's National AIDS and STI Control Program (NASCOP) and Division of HIS conducted assessments that identified software and grouped them as; Patient Management Software(e.g., $\quad$ C-PADComprehensive Patient Application Data base, OpenMRSOpen Medical Records Software, IQ-CARE and EDPMS), Hospital HMIS Software /ERP Systems, Data Collection and Reporting Software( e.g., C-PAD, OpenMRS, IQCARE, EPI-INFO), Data Analysis Software, Administration/Management Software, External Systems",

\section{$>$ Statement of The Problem}

Electronic Medical Records (EMR) software are basically, designed to facilitate patient data management in health facilities. However, Kenya's Standards and Guidelines for Electronic Medical Record Systems, 2010, revealed that harnessing quality information has been a challenge both in the public and private sectors. It reveals assessments done that identified EMR or patient management software specifically designed to support chronic HIV care as; C-PAD, OpenMRS, IQ-CARE, and EDPMS. Despite Ministry of Health in Kenya goal to strengthen delivery of health care through use of electronic medical record (EMR) systems as a key strategy to improve health records management and quality of patient care, certain facilities within Kisumu County have not yet adopted EMRs. The County has a total of 234 functional health facilities out of which only 57 (24\%) have adopted EMR. (Kenya Health Information system 2018). This shows that the level of EMR adoption in the county is still very low. However, new EMR software currently, are being availed in the market by vendors or implementers with various features. It is for this reason that the researcher sought to examine EMR software types in use within Kisumu County. 


\section{Specific Objectives}

- To examine Electronic Medical Records software types in use within Kisumu County

\section{Research Questions}

- What types of Electronic Medical Records software are in use within Kisumu County?

\section{LITERATURE REVIEW}

\section{> Introduction}

Globally, Health Information Systems (HIS) and technologies are being used increasingly and are seen as a way to increase hospital efficiency and the quality of patient care. HIS enable healthcare providers to document relevant patient information and provide efficient and improved healthcare services. Healthcare organizations achieve these outcomes through use of HIS such as Electronic Medical Records (EMR), Computer Based Patient Records (CBPR), Automated Health Records (AHR) and Electronic Patient Records (EPR) all of which enable patient information to be recorded electronically. The uptake of EMR systems throughout the world has occurred at different rates, with developing countries tending to lag behind more developed nations (Hasanain, Vallmur \& Clark 2014)

Electronic Medical Records (EMRs) are computerized medical information systems that collect, store and display patient information. They are a means to create legible and organized recordings and to access clinical information about individual patients (Boonstra \& Broekhuis 2010).

Benefits of using EMR have been documented mostly in high-income countries and include improved quality of care and high levels of client satisfaction, faster retrieval of client records and avoidance of missing folders, improved documentation, enhancement of within-facility healthcare coordination, improved efficiency and convenience in care provision, reduction in staff time spent on specific paperbased administrative tasks, reduction in number of duplicate diagnostic tests ordered, and improvement in patient safety and health outcomes (Gyamfi, Mensah, Oduro, Donkor \& Mock, 2017)

\section{Electronic Medical Records Software types}

The search yielded 147 publications of which 21papers from 15 sub-Saharan African countries documented the use of EHR in Africa and were reviewed. About $91 \%$ reported use of Open Source healthcare software, with OpenMRS being the most widely used. Most reports were from HIV related health centers. Barriers to adoption of EHRs include high cost of procurement and maintenance, poor network infrastructure and lack of comfort among health workers with electronic medical records.
Conclusion: There has been an increase in the use of EHRs in sub-Saharan Africa, largely driven by utilization by HIV treatment programs. Penetration is still however very low The search yielded 147 publications of which 21papers from 15 sub-Saharan African countries documented the use of EHR in Africa and were reviewed. About $91 \%$ reported use of Open Source healthcare software, with OpenMRS being the most widely used. Most reports were from HIV related health centers. Barriers to adoption of EHRs include high cost of procurement and maintenance, poor network infrastructure and lack of comfort among health workers with electronic medical records.

Conclusion: There has been an increase in the use of EHRs in sub-Saharan Africa, largely driven by utilization by HIV treatment programs. Penetration is still however very low The implementation of Clinical.

Information Systems has been identified as an important compo-nent of improving health care, and the degree of adoption of tech-nologies in health care has been shown to correlate with reduction of complications and mortalities in hospitals[20-22]. Although African

Akanbi et al., (2012), adds that "the degree of adoption of technologies in health care has been shown to correlate with reduction in mortalities in hospitalities. Most EHRs are sustained by funding from international partnerships raising questions about the sustainability of these systems by the host institutions. Emergence of Open Source Software has however enhanced the opportunity of several African countries to gain access to EHRs. About 91\% reported use of Open Source healthcare software, with Open MRS being the most widely used. Most reports were from HIV related health centers.".

Kenya in the year 2010 assessed the functionality of existing EMR systems implemented in Kenya through National AIDS and STI Control Program (NASCOP) and grouped the software as: Patient Management Software, Patient Application Data base, HMIS Software, Data Collection and Reporting Software, Data Analysis Software, Administration/Management Software, External Systems (Kang'a et. al., 2017)

\section{METHODOLOGY}

\section{Introduction}

This research adopted descriptive research design as it was useful in collecting data that described EMR software types available. About 163 study subjects from three Sub Counties responded to questionnaires administered for data collection. 


\section{RESULTS AND DISCUSSION}

\section{Introduction}

This section presents results and analysis of data collected based on study objectives.

Response rate

This included facility profile and response rate of respondents in Kisumu County

\begin{tabular}{|c|c|c|c|}
\hline & \multicolumn{3}{|c|}{ Questionnaire for EMR users } \\
\hline Sub County & Intended Questionnaires & Returned & Response Rate (\%) \\
\hline Kisumu Central & 142 & 113 & $80 \%$ \\
\hline Kisumu East & 35 & 29 & $83 \%$ \\
\hline Muhoroni & 50 & 21 & $42 \%$ \\
\hline Total & 227 & 163 & $71.8 \%$ \\
\hline
\end{tabular}

Table 1:- Response rate for EMR users

The researcher intended to reach a sample size of 227 respondents. However, only 163 out of 227 (71.8\%) questionnaires were received from EMR software users. This was mainly staff rotation/transfers affecting EMR sites supported by Comprehensive Care Clinic partners/NGO and time constraints.

\section{Study Objectives}

This study focused on study findings derived from objectives of the study.
- Electronic Medical Records software types in use within Kisumu County

The researcher found various kind of EMR software in use among sampled facilities within Kisumu County; Open MRS, Kenya EMR, IQ Care, E-Hospital, Funsoft and SYHOS. However, Open MRS, Kenya EMR and IQ Care software were found to be implemented in Comprehensive Care Clinics (CCC) for HIV Care and Treatment while EHospital, Funsoft and SYHOS are implemented in general outpatient departments.

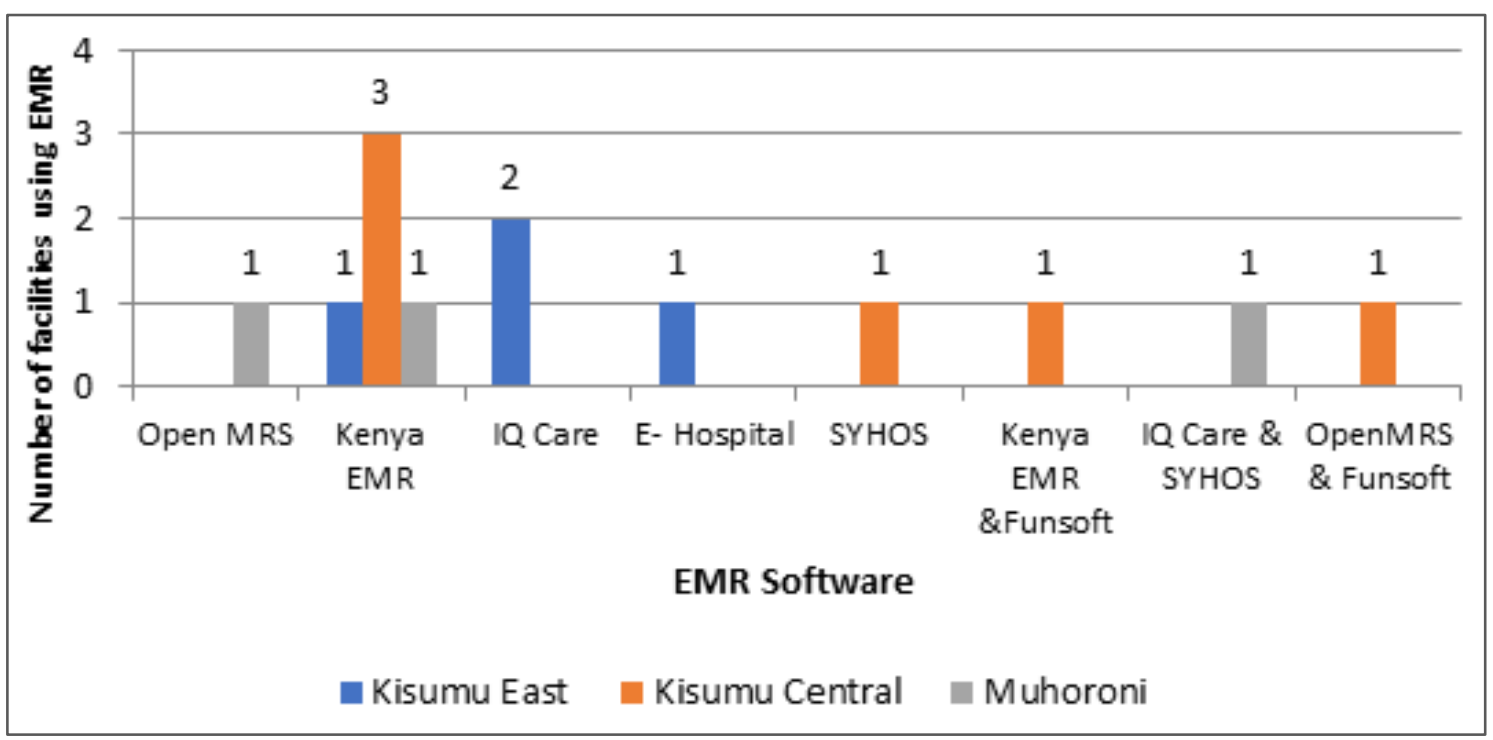

Fig 1:- Distribution of EMR Software in use per Sub County

Figure 1, shows number of health facilities from different Sub Counties using varied EMR Software. Kenya EMR software was predominantly in use in Kisumu Central Sub County compared to other health facilities sampled. However, most sites in Kisumu Central had two kinds of software in use for patient management and one health facility in Muhoroni Sub County using both IQ Care and SYHOS. 


\begin{tabular}{lccc}
\hline & \multicolumn{2}{c}{ EMR IMPLEMENTATION TYPE } & \\
Sub County & POC & HYBRID & RDE \\
\hline Kisumu East & $50 \%$ & $0 \%$ & $50 \%$ \\
Kisumu Central & $50 \%$ & $38 \%$ & $13 \%$ \\
Muhoroni & $25 \%$ & $25 \%$ & $50 \%$ \\
\hline
\end{tabular}

Table 2:- EMR Implementation type (design type)

Majority of health facilities that were using Funsoft, SYHOS and E-Hospital were implementing Point of Care as the software features are majorly designed to facilitate POC as opposed to Retrospective Data Entry (RDE). However, Open MRS, Kenya EMR and IQ care software are designed to facilitate POC, RDE or HYBRID (mixture of POC and RDE). Averagely, $42 \%$ of respondents were found to be implementing POC, followed by RDE (37\%) while $32 \%$ were implementing hybrid EMR model (mixture of Point of Care and Retrospective Data Entry). About 25\% staff in Muhoroni Sub County using the EMR as Point of Care (POC). This may be related to challenges regarding EMR implementation; likely to arise from technical/infrastructure issues and organizational challenges like, staffing levels, staff attitude and knowledge gap as well as support from top leadership.

\section{CONCLUSION AND RECOMMENDATION}

This study examined various software types in Kisumu County; OpenMRS, Kenya EMR, IQ Care, EHospital, Funsoft and SYHOS. However, Kenya EMR software was predominantly used by health care providers as opposed to other software probably due to the software features and support provided by implementing partners supporting HIV care and treatment.

It was also noted that Kisumu East (50\%) and Central Sub Counties $(50 \%)$ were implementing POC. This may be related to challenges regarding EMR implementation; likely to arise from technical/infrastructure issues and organizational challenges like, staffing levels, staff attitude and knowledge gap as well as support from top leadership.

\section{A. Conclusion}

Newer products are being availed in the market by vendors/implementers, therefore there is need to explore issues surrounding EMR to determine EMR software challenges regarding their implementation

\section{B. Recommendations}

Health facilities should develop strategic plans to provide direction to health facilities and communicate future aspirations of the facilities including EMR initiatives. Members of an organization should participate in use of EMRs and decision-making regarding adoption of EMRs.

\section{REFERENCES}

[1]. Akanbi, M. O., Ocheke, A. N., Agaba, P. A., Daniyam, C. A., Agaba, E. I., Okeke, E. N., \& Ukoli, C. O. (2012). Use of electronic health records in subSaharan Africa: progress and challenges. Journal of Medicine in the Tropics, 14(1), 1. Retrieved from https://www.ncbi.nlm.nih.gov

[2]. Boonstra, A., \& Broekhuis, M. (2010). Barriers to the acceptance of electronic medical recors by physicians from systematic review to taxonomy and interventions. BMC health services research, 10(1), 231.

[3]. Cowan, J. C., Curran, B., Dawoud, K., De Volder, D., Demestichas, D., Desa, K., ... \& Dolan, C. D. (2019). Untitled-International Institute of Informatics and Systemics.

[4]. Gyamfi, A., Mensah, K. A., Oduro, G., Donkor, P., \& Mock, C. N. (2017). Barriers and facilitators to Electronic Medical Records usage in the Emergency Centre at Komfo Anokye Teaching Hospital, KumasiGhana. African Journal of Emergency Medicine, 7(4), 177-182

[5]. Hasanain, R., Vallmuur, K., \& Clark, M. (2014). Progress and Challenges in the implementation of Electronic Medical Records in Saudi Arabia: a systematic review. Health Informatics- An International Journal, 3(2).

[6]. Kang'a, S., Puttkammer, N., Wanyee, S., Kimanga, D., Madrano, J., Muthee, V., \& Kwach, J. (2017). A national standards-based assessment on functionality of electronic medical records systems used in Kenyan public-Sector health facilities. International journal of medical informatics, 97, 68-75.

[7]. Kenya, D. H. (2018). Kenya Health Information System.

[8]. Marques, A., Oliveira, T., Dias, S. S., \& Martins, M. F. (2011). Medical records system adoption in European hospitals. Electronic Journal of Information Systems Evaluation, 14(1), 89. 